\title{
"The comparison of postoperative pain severity in different types of hospitals in the eastern Poland"
}

\author{
Borys M., Zyzak K., Wróblewski K., Piwowarczyk P., Czuczwar M. \\ Medical University of Lublin, II Dept of Anaesthesiology \& Intensive Care, Lublin, Poland
}

Aims and goals:

Pain assessment and treatment still does not cover all patients requirements. Many patients suffer from severe pain in postoperative period.

The main goal of the study was the assessment of pain severity among patients from different types of hospitals (primary, secondary and tertiary centers) in the similar types of surgeries. The other goals were if there was any difference in pain severity associated with anaesthesia techniques and type of surgery.

\section{Methods:}

The prospective, observational study. The VAS (visual-analogue scale) was used to measure pain severity 4 times on the day of surgery (at $0-4$, 4-8, 8-12, 24 hour). Demographic data, a types of hospital, surgery and anaesthesia were also collected.

Only adults who were able to note their pain severity participated in the study. VAS was not received from the patients anesthetized under local technique with or without sedation.

The analysis of variances (ANOVA) and t-test were used to assess the statistics. Contingency tables were utilized for demographics. All measurements were done with Statistica 12.5 (Stat Soft. Inc., Tulsa, USA)

\begin{tabular}{|l|l|l|l|l|l|}
\hline & Number & Percent & Mean age & Height $[\mathrm{cm}]$ & Weight $[\mathrm{kg}]$ \\
\hline Female & 269 & 47.8 & 52.78 & $162.42(161.10-163.74)$ & $71.82(69.93-73.73)$ \\
\hline Male & 293 & 52.2 & 50.78 & $174.10(173.06-175.14)$ & $82.68(80.54-84.82)$ \\
\hline overall & 562 & 100 & 51.74 & $168.51(167.55-169.47)$ & $77.49(75.98-78.99)$ \\
\hline
\end{tabular}

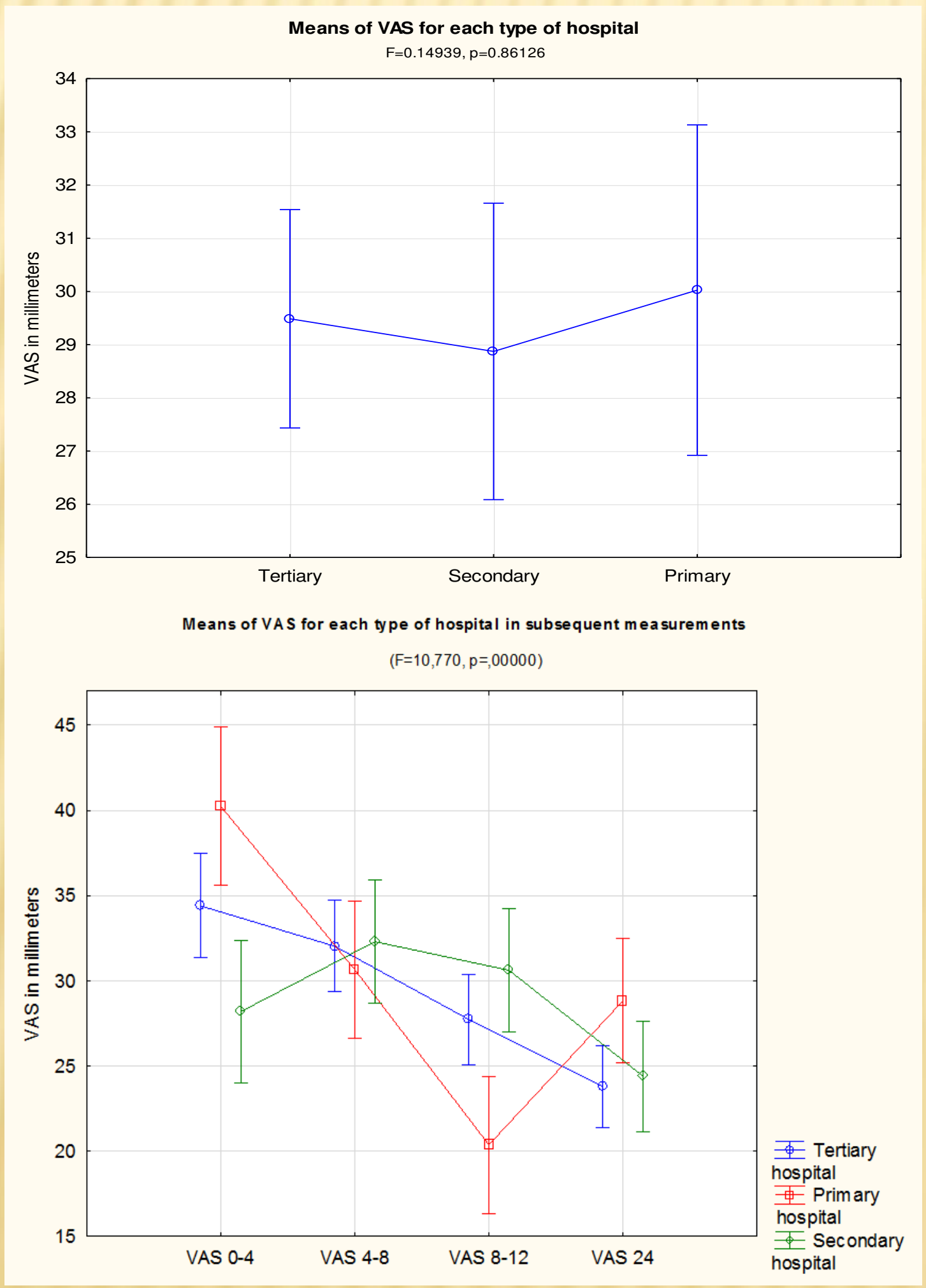

Acute pain assessment tools: let us move beyond simple pain ratings. Current Opinion in Acute pain assessment tools: let us move beyond simple pain rating
Anaesthesiology: October 2015 - Volume 28 - Issue 5 - p 565-569

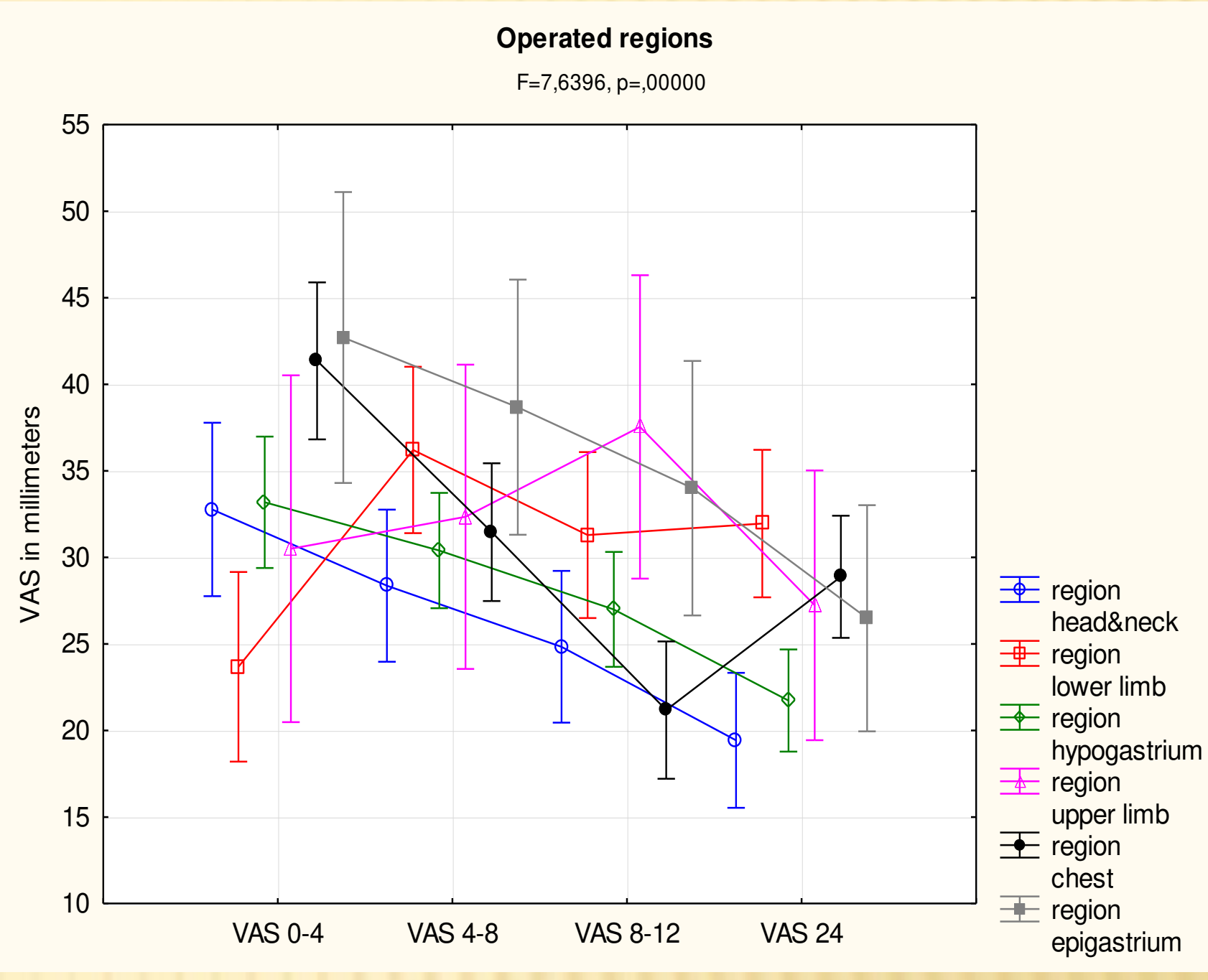

Results:

The study was conducted from November 2015 to June 2016 in 7 hospitals in the south-eastern Poland : 2 university, 2 secondary and 3 primary hospitals. VAS forms were collected from 565 patients .

The number of patients from tertiary hospitals was 284, from secondary hospitals 154 and primary ones was 124 . There was no statistical difference in general VAS value between types of hospitals.

The average values of VAS: tertiary hospitals 29.49 (27.43-31.54), secondary hospitals $28.87(26.08-31.66)$ and primary hospitals 30.02 (26.91-33.13); $\mathrm{F}=0.149, \mathrm{p}=0.861$.

However, there was a significant difference between primary and secondary hospitals at 4 hour $(\mathrm{F}=7,32, \mathrm{p}=0.000727)$. 0 - 4 VAS values: primary hospitals 40.25 (35.62-44.88), secondary hospitals 28.12 (24.02-32.34). Moreover, the difference was found between primary hospitals and both secondary and tertiary hospitals ( $\mathrm{F}=7.29$, $\mathrm{p}=0.000745$ ). The mean $8-12$ VAS values were: primary hospitals 20.37 (16.34-24.39), secondary hospitals 30.62 (27.0-34.23), tertiary hospitals 27.73 (25.06-30.38).

According to the operated region, there was a significant difference at 4 hour between lower limb surgery and the epigastric region and chest surgeries $(\mathrm{F}=5.77, \mathrm{p}=0.000033)$. 0-4 VAS values: lower limb 23.68 (18.20- 29.15), chest 41.35 (36.82-45.89), epigastrium 42.70 (34.3051.10).

At 8-12 hour after surgery there was a significant difference between chest and lower limb surgeries $(F=4.28, p=0,00079)$. VAS values: chest 21.18 (17.21-25.15), lower limb 31.28 (26.49-36.08).

At 24 hour there was a significant difference between lower limb and either head and neck or epigastrium surgeries. $(\mathrm{F}=5.62, \mathrm{p}=0,000046)$. 24 hour VAS: lower limb 31.95 (27.69-36.21), head and neck 19.43 (15.53-23.32), epigastrium 26.48 (19.95-33.02).

There was only found a significant difference between general and subarachnoid types of anaesthesia at 4 hour $(\mathrm{F}=6.53, \mathrm{p}=0,0000002)$. The VAS values: general 39.41 (36.66-42.17), subarachnoid 25.47 (20.68-30.26).

Conclusions: the type of hospital may influence patients' pain severity, but more research needs to be done. 